\title{
Enhanced Photocatalytic Activity of 3,4,9,10-Perylenetetracarboxylic Diimide Modified Titanium Dioxide Under Visible Light Irradiation
}

\author{
Ji Won Kim, ${ }^{*}$ Hee Sung Kim, ${ }^{\dagger}$ Kook Hyun Yu, Akira Fujishima, ${ }^{\dagger}$ and Young Soon Kim \\ Department of Chemistry, Dongguk University, Seoul 100-715, Korea. *E-mail: jwkim1234@dongguk.edu \\ †SamMyung Bio Chem Co., Ltd., Chungbuk 363-952, Korea \\ ${ }^{\ddagger}$ Kanagawa Academy of Science and Technology, 3-2-1 Sakado, Takatsu-ku, Kawasaki-shi, Kanagawa 213-0012, Japan \\ Received August 9, 2010, Accepted August 18, 2010
}

\begin{abstract}
A method to improve the photocatalytic activity of titanium dioxide by modification with a sensitizer and a metal oxide is proposed. To achieve this goal, we used metal oxides as dopants. In particular, $\mathrm{CaWO}_{4}$ and $\mathrm{Gd}_{2} \mathrm{O}_{2} \mathrm{~S}$ : Tb were used because their $2.6 \mathrm{eV}$ and $2.2 \mathrm{eV}$ band gap energy and optical properties have a large positive effect on photocatalysis. The improvement in the photocatalytic activity of $\mathrm{TiO}_{2}$ modified with $\mathrm{Gd}_{2} \mathrm{O}_{2} \mathrm{~S}$ : $\mathrm{Tb}$ under ultraviolet light irradiation is described in a previous study. The present work focuses on the sensitization of metal oxide-modified $\mathrm{TiO}_{2}$. Having observed the ultraviolet-visible absorption spectra of 3,4,9,10-Perylenetetracarboxylic diimide in the wide visible-light region from $400 \mathrm{~nm}$ to $650 \mathrm{~nm}$ and the broad peaks in its photoluminescence spectra at $695 \mathrm{~nm}$ and $717 \mathrm{~nm}$, we decided to use this perylene dye to sensitize modified $\mathrm{TiO}_{2}$ to enhance its activity as a visible-light harvesting photocatalyst. We also explored the positive effects thin-film surface changes stemming from ultraviolet pre-treatment have on photocatalytic activity. Finally, we subjected several metal oxide-modified $\mathrm{TiO}_{2}$ products sensitized by the perylene dye to ultraviolet pre-treatment, obtaining the most active photocatalysts.
\end{abstract}

Key Words: Titanium dioxide, $\mathrm{TiO}_{2}$, Photocatalyst, Sensitizer, 3,4,9,10-Perylenetetracarboxylic diimide

\section{Introduction}

With increasing calls for a cleaner environment, photocatalytic applications used by industry are attracting renewed interest. For example, many concerned scientists see decomposition of organic pollutants by photocatalysis as one way to achieve the goal of an eco-friendly economy. And another advantage of photocatalytic technology is that it can be very cost effective because sunlight can be used as an energy source. ${ }^{1-5}$

Semiconductor materials, such as $\mathrm{TiO}_{2}, \mathrm{ZnO}, \mathrm{SnO}_{2}, \mathrm{CdS}$, $\mathrm{GaP}$, and $\mathrm{WO}_{3}$, are applied in photocatalysis. Among them, $\mathrm{TiO}_{2}$ is the most popular for this purpose because it is nontoxic, chemically stable, inexpensive, and very efficient. In fact, $\mathrm{TiO}_{2}$ photocatalysts are frequently employed in environmental cleanups. However, their photocatalytic activity is restricted by the wide band gap of $\mathrm{TiO}_{2}(3.03 \mathrm{eV}$ for rutile and $3.18 \mathrm{eV}$ for anatase) and fast electron-hole recombination. ${ }^{6-9} \mathrm{TiO}_{2}$ can absorb only about $3 \%-4 \%$ of solar light in the ultraviolet, or UV, region. To increase the amount of visible light (to about $45 \%$ ) $\mathrm{TiO}_{2}$ absorbs from the sun, researchers are working on the development of $\mathrm{TiO}_{2}$ visible-light harvesting photocatalysts. ${ }^{10}$

The visible-light sensitivity of $\mathrm{TiO}_{2-\mathrm{x}} \mathrm{N}_{\mathrm{x}}$ is a result of the narrowing of the band gap by mixing the $2 p$ states of nitrogen and $2 p$ states of oxygen. ${ }^{5,11}$ In semiconductor photocatalysis the visible or UV light forms electrons and holes in the semiconductor material, which are essential to the catalytic reactions. We investigated metal-oxide doping because of its ability to narrow the band gap. Under UV light irradiation, the photocatalytic activity of metal oxide-modified $\mathrm{TiO}_{2}$, or $\mathrm{TiO}_{2} /$ metal oxide, was $13 \%$ - $35 \%$ higher than that of $\mathrm{TiO}_{2}(\mathrm{P} 25)$ under UV light irradiation; however, the activity was still low under visible light $(\geq 400 \mathrm{~nm})$ irradiation. This makes practical use difficult. We addressed these drawbacks by sensitizing $\mathrm{TiO}_{2} /$ metal oxide to obtain a visible-light harvesting photocatalyst. The fluorescent dye 3,4,9,10-Perylenetetracarboxylic diimide or PTCDI was used as the sensitizer because of its high thermal stability and photostability. PTCDI is used extensively as an industrial pigment, and its derivatives are effective n-type organic semiconductors with various applications. For example, they are used as tunable laser dyes, light-harvesting materials, transistors, solar cells, and optoelectronic devices, such as light-emitting diodes, because of their unique optical and electrochemical properties. $^{12,13}$

In the present work, we report the enhanced photocatalytic activity under visible-light irradiation of metal oxide-modified $\mathrm{TiO}_{2}$ sensitized with PTCDI. We also propose an efficient method for the preparation of photocatalyst thin film.

\section{Experimental}

Material. Titanium dioxide powder (Degussa, P25) was used as the photocatalyst base material. It was composed of $80 \%$ anatase and $20 \%$ rutile, with a specific surface area of BET $60 \pm$ $5 \mathrm{~m}^{2} \mathrm{~g}^{-1}$. The metal oxides were $\mathrm{Ag}_{2} \mathrm{O}, \mathrm{CaWO}_{4}, \mathrm{Fe}_{2} \mathrm{O}_{3}$ (Aldrich Co.,USA; analytic reagent grade), and $\mathrm{Gd}_{2} \mathrm{O}_{2} \mathrm{~S}$ :Tb. 3,4,9,10Perylenetetracarboxylic diimide (Tokyo Chemical Industry Co., first grade reagent) was used as the sensitizer. Ethanol (Wako Chemical, USA) was used as the dispersion medium. The thinfilm substrate was a petri dish $(8.6 \mathrm{~cm} \times 1.7 \mathrm{~cm})$. Methylene blue and formaldehyde (Aldrich Co., first grade reagent) were used as is. Water-borne polyurethane dispersion (SamMyung Biochem Co., Korea, pH 7, M.W 8000, MTU-06) was used as binder.

We used a furnace (Muffle-1125, Dongyang Science Co.) to prepare the $\mathrm{TiO}_{2} /$ metal oxide. The thin films were prepared by spray coating (NCF 035CC, Air Maker, Korea) under a pre- 
ssure of 7 bar. The specific surface area was measured by BET (ASAP 2020, Micromeritics Ins., USA). The absorbance of methylene blue was investigated by ultraviolet-visible, or UV-vis, spectrophotometry (DT 1000 CE, Ocean optics Inc., USA), and the decomposition rate of the formaldehyde was measured by gas chromatography (HP 6890 Series, USA).

Sensitization of $\mathrm{TiO}_{2}$ thin films. $0.013 \mathrm{~g}$ of PTCDI was added to $\mathrm{TiO}_{2}$ and a Vortex mixer was used to mix them for 5 minutes. At this point the specific surface area ratio of the PTCDI to $\mathrm{TiO}_{2}$ was BET 5\% - 10\%. Distilled water $(60 \mathrm{~mL})$ as solvent was added to the mixture, which was stirred at room temperature for $16 \mathrm{hrs}$. The water was removed by drying the mixture for $22 \mathrm{hr}$ in an oven (HST501VS, HST Co.) at $120{ }^{\circ} \mathrm{C}$. Preparation of the $\mathrm{TiO}_{2} /$ metal oxide was according to our previous study, ${ }^{14}$ with $0.005 \mathrm{~g}$ of metal oxide added to $0.04 \mathrm{~g}\left(5 \times 10^{-4}\right.$ $\mathrm{mol}$ ) of $\mathrm{TiO}_{2}$. Using the identical procedure described above, we sensitized the $\mathrm{TiO}_{2} /$ metal oxide and calcinated it at $400{ }^{\circ} \mathrm{C}$ for 45 minutes.

Ethanol $(15 \mathrm{~mL})$ was added to each of the powder mixtures as a dispersant and then water-born polyurethane was added to the dispersed solutions, after which they were sonicated for $1 \mathrm{hr}$. The water-born polyurethane acted as a binder, immobilizing $\mathrm{TiO}_{2}$ particles and preventing the degradation of photocatalytic potential. Thin films were prepared from the dispersed solutions by a spray coating technique.

Photocatalytic activity measurements. The photocatalytic activity of each sample, including the PTCDI-modified $\mathrm{TiO}_{2}$, was determined by the change in absorbance. The change in absorbance was determined by measuring the percent of decoloration of aqueous methylene blue solution by photocatalysis. The photocatalyst thin films were coated with $40 \mu \mathrm{M}$ of aqueous methylene blue solution and placed under a UV cut-off filter $(\mathrm{N}-169(400 \mathrm{~nm} \leq))$. Visible light was irradiated with two fluorescent light lamps $\left(20 \mathrm{~W}, \geq 380 \mathrm{~nm}, 9 \mathrm{~W} / \mathrm{m}^{2}\right)$ placed at a distance of $20 \mathrm{~cm}$. During a $5-\mathrm{hr}$ period, we took absorbance measurements once an hour. The photodegradation of formaldehyde was measured by the method described earlier. ${ }^{14} \mathrm{We}$ added $0.1 \mathrm{M}$ formaldehyde to the photocatalyst thin films and measured its decomposition rate during a 2-hr period of visible-light irradiation.

Characterization of thin film transmittance and photocatalytic activity. Photocatalytic activity and transmittance were measured for each of the thin-film coatings, which were numbered from 1 through 20. The measurements, by UV-vis spectrophotometry, discolored the methylene blue.

Characterization of UV pre-treatment effect. UV pre-treatment was started when the photocatalytic activity was at its highest level. An earlier investigation of $5 \mathrm{hr}$ through $28 \mathrm{hr}$ of activity showed the 16th $\mathrm{hr}$ to be optimum. UV pre-treatment was carried out using two black lamps (Sankyo denki black light blue $\left.\left(20 \mathrm{~W}, 352 \mathrm{~nm}, 16 \mathrm{~W} / \mathrm{m}^{2}\right)\right)$ placed at a distance of $15 \mathrm{~cm}$.

Characterization of photoluminescence. Measurement of the photoluminescence of the sensitizer-modified $\mathrm{TiO}_{2} /$ metal oxide was performed as described previously. ${ }^{14}$

\section{Results and Discussion}

Absorption spectrum of 3,4,9,10-perylenetetracarboxylic

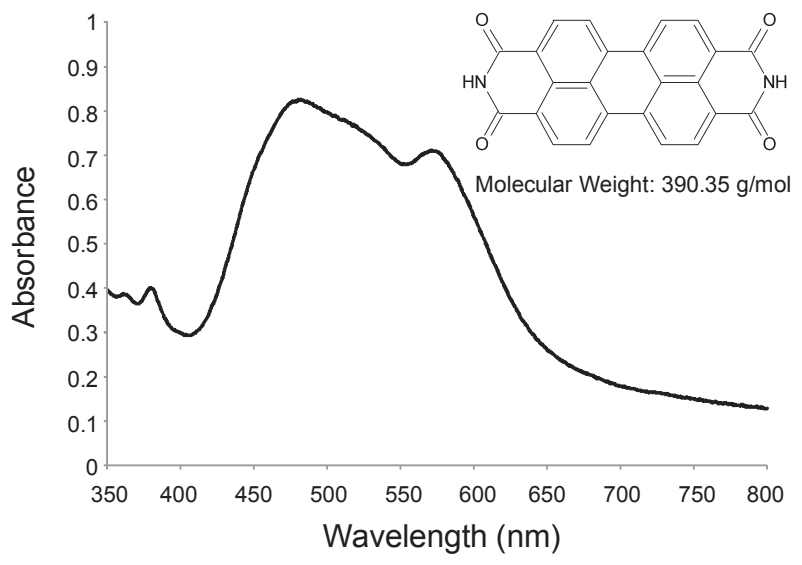

Figure 1. The chemical structure and UV-vis spectrum of 3,4,9,10perylenetetracarboxylic diimide.

diimide. The level of photocatalytic activity of a photocatalyst depends on the number of excited electrons created by the energy supplied. If the intensity of the supplied energy equals an intensity within the visible-light region, the photocatalyst might be active under visible-light irradiation. Sensitization can improve performance by expanding photocatalytic activity to the visible-light region. The chemical structure and UV-vis spectra of the sensitizer, PTCDI, are shown in Figure 1. Since the UV-vis absorption spectra of PTCDI are in the wide visiblelight region (400 nm - $650 \mathrm{~nm}$ ), we expected sensitization to enhance the activity of visible-light harvesting photocatalysts. Moreover, PTCDI displays a high degree of photostability, making it even more effective.

Thin film transmittance and methylene blue decoloration rate. The most important consideration in the preparation of photocatalyst thin films is how to efficiently optimized the photocatalytic activity. Although $\mathrm{TiO}_{2}$ photocatalyst powders have identical properties, significant differences in photocatalytic activity arise among them because of variations in the amount of $\mathrm{TiO}_{2}$ dispersed in solution or the thickness of their thin films. Various methods are used to prepare thin films, including spray coating, dip coating and spin coating. In the present study, we used spray coating, because it is effective regardless of the characteristics of the thin-film substrate. A coating speed of $2.0 \pm$ $0.05 \mathrm{~cm} / \mathrm{sec}$ was adhered to as closely as possible. Figure 2 confirms that this coating technique optimized the thin-film photocatalytic activity.

Although binder in the dispersed solution decreased thin-film transmittance, the decoloration rate of methylene blue indicates that photocatalytic activity did increased (Figure 2). This result is explained by the fact that photocatalysis occurs at the surface of the thin films. Increasing the number of coatings enhances photocatalytic activity because of the dense distribution of photocatalyst particles (Figure 3). But, excessive coating number cause the disturbance of photocatalytic activity by increased binder amount. For the reason, Eight coatings showed the highest methylene-blue decoloration rate, and this number maintained the transmittance of the thin films at more than $85 \%$.

UV pre-treatment effect. The degree of unsaturation of surface bonds effects the photohydrophilicity of thin films. UV irradiation can produce hydroxyl groups, promoting photo- 


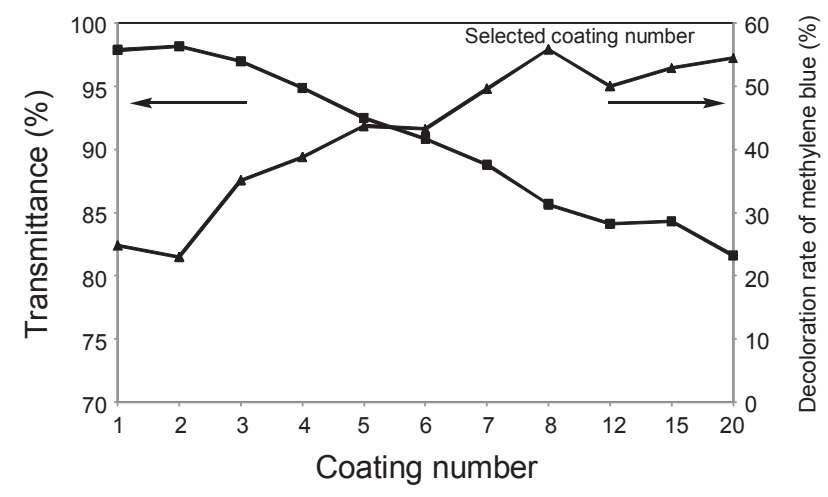

Figure 2. Relations with thin film transmittance and decoloration rate of methylene blue. Increasing the number of coating, decoloration rate of methylene blue means photocatalytic activity increase to a certain portion compared with decreased transmittance of thin film.

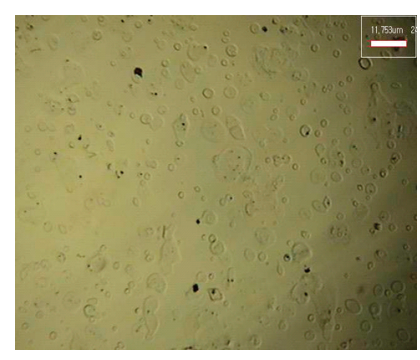

(A)

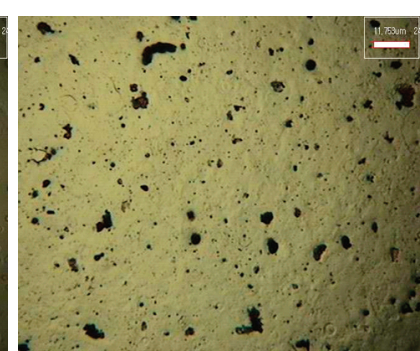

(B)
Figure 3. Micrograph of thin film surface (Video microscope (SV-35, Sometech Co. $(\times 600))$ ). (A) Characterized $\mathrm{TiO}_{2}$ thin film with coating number 1 , (B) Characterized $\mathrm{TiO}_{2}$ thin film with coating number 8 . Increasing the number of coating, distribution of the $\mathrm{TiO}_{2}$ photocatalysts particles is densely packed.

hydrophilicity. This change on the thin-film surface increases the number of water molecules absorbed on the surface. The UV-vis spectra, which shifted to the shorter wavelength after UV irradiation, indicated the photohydrophilicity of the $\mathrm{TiO}_{2}$ thin films. ${ }^{15}$ This confirmed the positive effect UV pre-treatment had on photocatalytic activity. Also, the increased rate of decoloration of methylene blue by UV pre-treatment of the $\mathrm{TiO}_{2} /$ metal oxide thin films was confirmed, showing that UV pretreatment improved their photocatalytic activity. For $\mathrm{TiO}_{2}(\mathrm{P} 25)$, the decoloration of methylene blue increased by 24.26 percentage points, from $4.88 \%$ to $29.14 \%$ as a result of UV pre-treatment. And the photocatalytic activity of the other $\mathrm{TiO}_{2} / \mathrm{metal}$ oxide thin films increased by $4.70-9.06$ percentage points (Table 1). The activity of the PTCDI-modified $\mathrm{TiO}_{2}$ increased by 4.77 percentage points, from $36.73 \%$ to $41.50 \%$ as a result of UV pre-treatment. And UV pre-treatment increased the activity of the PTCDI-modified $\mathrm{TiO}_{2} /$ metal oxide by 4.70 - 14.20 percentage points (Table 2). However, after UV light irradiation, the photocatalytic activity of the $\mathrm{TiO}_{2} / \mathrm{Gd}_{2} \mathrm{O}_{2} \mathrm{~S}$ : Tb decreased despite PTCDI modification. Perhaps, this phenomenon can be explained by the disturbance created between alternations in the characteristics of the thin-film surface and the transition of an electron from ${ }^{5} \mathrm{D}_{3}$ state to the conduction band or to the ${ }^{5} \mathrm{D}_{4}$ level in the identical anionic environments of the $\mathrm{Tb}^{3+}$ ion. ${ }^{14}$ Further theoretical study is necessary to elucidate the reason for the lower photocatalytic activity of $\mathrm{TiO}_{2} / \mathrm{Gd}_{2} \mathrm{O}_{2} \mathrm{~S}$ : Tb after UV pre-treatment.

Photodecoloration of methylene blue solution. Before measuring photocatalytic activity, we compared the initial absorbance of the aqueous methlyene blue solution with its final absorbance after photocatalysis. Next, we compared the results of the photodecoloration rate of methylene blue (\%) shown in Table 1 and Table 2 . Table 1 shows the photocatalytic activity of $\mathrm{TiO}_{2}(\mathrm{P} 25)$ and $\mathrm{TiO}_{2} /$ metal oxide. $\mathrm{TiO}_{2}(\mathrm{P} 25)$ was nearly photocatalytically inactive, but the activity of $\mathrm{TiO}_{2} /$ metal oxide increased by 23.35 - 46.47 percentage points under visible light. In particular, because of their lower band gap energy $(2.6 \mathrm{eV}$ and $2.2 \mathrm{eV}$ ) $\mathrm{CaWO}_{4}$ and $\mathrm{Gd}_{2} \mathrm{O}_{2} \mathrm{~S}$ : Tb showed increased photocatalytic activity compared with the other metal oxides. In addition, the behavior of $\mathrm{Gd}_{2} \mathrm{O}_{2} \mathrm{~S}$ : Tb was greatly influenced by its optical properties, such as the transition of an electron from the ${ }^{5} \mathrm{D}_{3}$ state to the conduction band or to the ${ }^{5} \mathrm{D}_{4}$ level in the identical anionic environments of the $\mathrm{Tb}^{3+}$ ion. Table 2 shows the photocatalytic activity of $\mathrm{TiO}_{2}$ sensitized with PTCDI.

Table 1. Confirmation of the UV pre-treatment effect on metal-oxide modified $\mathrm{TiO}_{2}$ thin film

\begin{tabular}{|c|c|c|c|c|c|}
\hline \multirow[b]{2}{*}{ Sample } & \multicolumn{3}{|c|}{ Non UV pre-treatment } & \multicolumn{2}{|c|}{ UV pre-treatment } \\
\hline & $\begin{array}{l}\text { Photodecoloration of } \\
\text { MB by visible light } \\
\text { absorption (\%) }\end{array}$ & $\begin{array}{c}\text { 1st order rate constant } \\
\text { for photodecoloration } \\
\text { of } \mathrm{MB}(\%)\end{array}$ & $\begin{array}{c}\text { Photocatalytic } \\
\text { degradation of } \\
\text { formaldehyde by GC (\%) }\end{array}$ & $\begin{array}{l}\text { Photodecoloration of } \\
\text { MB by visible light } \\
\text { absorption }(\%)\end{array}$ & $\begin{array}{c}\text { 1st order rate constant } \\
\text { for photodecoloration } \\
\text { of } \mathrm{MB}(\%)\end{array}$ \\
\hline $\mathrm{TiO}_{2}(\mathrm{P} 25)$ & $4.88^{a}$ & $1.03^{b}$ & $2.29^{c}$ & $29.14^{a}$ & $6.69^{b}$ \\
\hline $\mathrm{TiO}_{2} / \mathrm{Ag}_{2} \mathrm{O}$ & $\begin{array}{c}+23.352 \\
28.23 \pm 0.41^{a}\end{array}$ & $\begin{array}{c}+5.522 \\
6.55 \pm 0.32^{b}\end{array}$ & $\begin{array}{r}+19.173 \\
21.46^{c}\end{array}$ & $\begin{array}{c}+8.155 \\
37.29 \pm 0.17^{a}\end{array}$ & $\begin{array}{c}+2.114 \\
8.80 \pm 0.23^{b}\end{array}$ \\
\hline $\mathrm{TiO}_{2} / \mathrm{Fe}_{2} \mathrm{O}_{3}$ & $\begin{array}{c}+29.257 \\
34.13 \pm 0.27^{a}\end{array}$ & $\begin{array}{c}+7.483 \\
8.51 \pm 0.26^{b}\end{array}$ & $\begin{array}{c}+16.568 \\
18.85^{c}\end{array}$ & $\begin{array}{c}+10.029 \\
39.16 \pm 0.21^{a}\end{array}$ & $\begin{array}{c}+2.537 \\
9.22 \pm 0.26^{b}\end{array}$ \\
\hline $\mathrm{TiO}_{2} / \mathrm{CaWO}_{4}$ & $\begin{array}{c}+39.541 \\
44.42 \pm 0.18^{a}\end{array}$ & $\begin{array}{c}+10.582 \\
11.61 \pm 0.21^{b}\end{array}$ & $\begin{array}{r}+11.872 \\
14.09^{c}\end{array}$ & $\begin{array}{c}+19.984 \\
49.12 \pm 0.32^{a}\end{array}$ & $\begin{array}{c}+5.913 \\
12.60 \pm 0.30^{b}\end{array}$ \\
\hline $\mathrm{TiO}_{2} / \mathrm{Gd}_{2} \mathrm{O}_{2} \mathrm{~S}: \mathrm{Tb}$ & $\begin{array}{c}+46.473 \\
51.35 \pm 0.34^{a}\end{array}$ & $\begin{array}{c}+11.249 \\
12.27 \pm 0.23^{b}\end{array}$ & $\begin{array}{c}+31.834 \\
34.09^{c}\end{array}$ & $\begin{array}{c}+14.144 \\
43.28 \pm 0.29^{a}\end{array}$ & $\begin{array}{c}+3.586 \\
10.27 \pm 0.35^{b}\end{array}$ \\
\hline
\end{tabular}

${ }^{a}$ Decrease in concentration (\%), ${ }^{b}$ Rate constant $\left(\times 10^{-2} \mathrm{M} \mathrm{hr}^{-1}\right),{ }^{c}$ Percentage of photocatalytic degradation (\%). 
Table 2. The enhanced photocatalytic activity of metal-oxide modified $\mathrm{TiO}_{2}$ after sensitization with 3,4,9,10-perylenetetracarboxylic diimide and confirmation of the UV pre-treatment effect

\begin{tabular}{|c|c|c|c|c|c|}
\hline \multirow[b]{2}{*}{ Sample } & \multicolumn{3}{|c|}{ Non UV pre-treatment } & \multicolumn{2}{|c|}{ UV pre-treatment } \\
\hline & $\begin{array}{l}\text { Photodecoloration of } \\
\text { MB by visible light } \\
\text { absorption (\%) }\end{array}$ & $\begin{array}{c}\text { 1st order rate constant } \\
\text { for photodecoloration } \\
\text { of } \mathrm{MB}(\%)\end{array}$ & $\begin{array}{c}\text { Photocatalytic } \\
\text { degradation of } \\
\text { formaldehyde by GC (\%) }\end{array}$ & $\begin{array}{l}\text { Photodecoloration of } \\
\text { MB by visible light } \\
\text { absorption (\%) }\end{array}$ & $\begin{array}{c}\text { 1st order rate constant } \\
\text { for photodecoloration } \\
\text { of } \mathrm{MB}(\%)\end{array}$ \\
\hline $\mathrm{TiO}_{2}(\mathrm{P} 25)$ & $36.73 \pm 0.35^{a}$ & $8 . \overline{7} 4^{b}$ & $20.57^{c}$ & $41.50 \pm 0.14^{a}$ & $10.50^{b}$ \\
\hline $\mathrm{TiO}_{2} / \mathrm{Ag}_{2} \mathrm{O}$ & $\begin{array}{c}+16.032 \\
52.76 \pm 0.43^{a}\end{array}$ & $\begin{array}{c}+6.542 \\
15.28 \pm 0.24^{b}\end{array}$ & $\begin{array}{l}+9.859 \\
30.42^{c}\end{array}$ & $\begin{array}{c}+25.461 \\
66.96 \pm 0.16^{a}\end{array}$ & $\begin{array}{c}+10.352 \\
20.85 \pm 0.32^{b}\end{array}$ \\
\hline $\mathrm{TiO}_{2} / \mathrm{Fe}_{2} \mathrm{O}_{3}$ & $\begin{array}{c}+17.876 \\
54.60 \pm 0.27^{a}\end{array}$ & $\begin{array}{c}+7.441 \\
16.18 \pm 0.18^{b}\end{array}$ & $\begin{array}{l}+4.873 \\
25.44^{c}\end{array}$ & $\begin{array}{c}+23.728 \\
65.22 \pm 0.30^{a}\end{array}$ & $\begin{array}{c}+9.313 \\
19.81 \pm 0.41^{b}\end{array}$ \\
\hline $\mathrm{TiO}_{2} / \mathrm{CaWO}_{4}$ & $\begin{array}{c}+13.471 \\
50.20 \pm 0.18^{a}\end{array}$ & $\begin{array}{c}+4.09 \\
12.83 \pm 0.19^{b}\end{array}$ & $\begin{array}{c}23.701 \\
44.27^{c}\end{array}$ & $\begin{array}{c}+13.406 \\
54.90 \pm 0.26^{a}\end{array}$ & $\begin{array}{c}+3.349 \\
13.84 \pm 0.21^{b}\end{array}$ \\
\hline $\mathrm{TiO}_{2} / \mathrm{Gd}_{2} \mathrm{O}_{2} \mathrm{~S}: \mathrm{Tb}$ & $\begin{array}{c}+22.290 \\
59.02 \pm 0.31^{a}\end{array}$ & $\begin{array}{c}+8.10 \\
16.84 \pm 0.29^{b}\end{array}$ & $\begin{array}{l}+19.255 \\
39.82^{c}\end{array}$ & $\begin{array}{c}+8.957 \\
50.45 \pm 0.26^{a}\end{array}$ & $\begin{array}{c}+2.374 \\
12.87 \pm 0.22^{b}\end{array}$ \\
\hline
\end{tabular}

${ }^{a}$ Decrease in concentration (\%), ${ }^{b}$ Rate constant $\left(\times 10^{-2} \mathrm{M} \mathrm{hr}^{-1}\right),{ }^{c}$ Percentage of photocatalytic degradation (\%).

Compared with Table 1, the photocatalytic activity of PTCDImodified $\mathrm{TiO}_{2}$ is 31.85 percentage points higher than that of $\mathrm{TiO}_{2}(\mathrm{P} 25)$. Furthermore, the activity of PTCDI-modified $\mathrm{TiO}_{2} /$ metal oxide increased by $7.67 \%-24.53 \%$ compared with that of $\mathrm{TiO}_{2} /$ metal oxide. These results represent an improvement in photocatalytic activity through PTCDI and metal-oxide modification. The UV-vis absorption spectra with PTCDI as sensitizer were observed in the wide visible-light region, and PTCDI properties, such as high fluorescence quantum yields in solution $\left(\Phi_{\mathrm{F}} \geq 95 \%\right)$, chemical inertness, and photochemical stability, had a positive influence on photocatalytic activity. ${ }^{16}$

Kinetics of photodecoloration for methylene blue through the 1st order rate constant. Table 1 and Table 2 give the firstorder rate constants for the photodecoloration of methylene blue. These results were obtained by calculating the correlation between the length of time of visible-light irradiation and the decreasing ratio of methylene blue. This correlation is described with the following equation, using the first-order rate con$\operatorname{stant}(\mathrm{k})$

$$
-\mathrm{k}=\ln \left(\mathrm{C} / \mathrm{C}_{0}\right) /\left(\mathrm{t}-\mathrm{t}_{0}\right)
$$

$\mathrm{C}_{0}$ is the initial concentration of methylene blue solution and $\mathrm{C}$ is its concentration after photocatalysis. $\mathrm{t}-\mathrm{t}_{0}$ is the time for photocatalysis to proceed. The first-order rate constant $(\mathrm{k})$ shows a linear relationship with the photodecoloration of methylene blue solution. This shows that the photocatalysis rate varies with the type of prepared thin film. In the case of non UV-pretreatment, each of the first-order rate constants of PTCDImodified $\mathrm{TiO}_{2}$ and PTCDI-modified $\mathrm{TiO}_{2} /$ metal oxide increased $8.74-16.84 \pm 0.29 \times 10^{-2} \mathrm{M} \mathrm{hr}^{-1}$ more than the rate constants of $\mathrm{TiO}_{2}(\mathrm{P} 25)$ and $\mathrm{TiO}_{2} /$ metal oxide increased, which was 1.03 $12.27 \pm 0.23 \times 10^{-2} \mathrm{M} \mathrm{hr}^{-1}$. And, the first-order rate constants of the UV pre-treated thin films showed more improvement than the constants of the non UV pre-treated thin films regardless of PTCDI sensitization. Finally, the present work shows that among the several metal oxides studied, $\mathrm{TiO}_{2} /$ metal oxide pretreated with UV and sensitized with PTCDI was the kinetically superior photocatalyst.

Photodegradation of formaldehyde. The photodegradation of formaldehyde was studied with reference to the "sick house syndrome," a phrase used to describe a situation in which the residents of a house become ill and their illness is linked to the house's air quality. Formaldehyde is often cited as one cause of such a malaise.

Our investigation was performed in the dark because of the low photostability of formaldehyde. The decomposition rates of formaldehyde were measured by gas chromatography. In the procedure, 13.2 M aqueous formaldehyde was diluted to $0.1 \mathrm{M}$ followed by photocatalytic degradation for $2 \mathrm{hrs}$ under visible-light irradiation. Table 1 shows the effect of UV pretreatment. The photodegradation of formaldehyde with $\mathrm{TiO}_{2}$ (P25) is negligible, 2.29\%. Photodegradation with $\mathrm{TiO}_{2} /$ metal oxide is $11.8 \%-31.8 \%$ higher than with $\mathrm{TiO}_{2}(\mathrm{P} 25)$. Sensitization of $\mathrm{TiO}_{2}(\mathrm{P} 25)$ with PTCDI increases photocatalytic degradation with $\mathrm{TiO}_{2}$ (P25) from $2.29 \%$ (Table 1) to $20.57 \%$ (Table 2), confirming the sensitizer effect. The photocatalytic activity of PTCDI-modified $\mathrm{TiO}_{2} /$ metal oxide is $5.73 \%-30.18 \%$ higher than that of $\mathrm{TiO}_{2} /$ metal oxide. These results show different tendencies with respect to the decoloration of methylene blue, but improved photocatalytic activity with $\mathrm{CaWO}_{4}, \mathrm{Gd}_{2} \mathrm{O}_{2} \mathrm{~S}$ : $\mathrm{Tb}$, and PTCDI is confirmed.

Photoluminescence spectra of sensitizer-modified $\mathrm{TiO}_{2}$ powders. Figure 4 shows the photoluminescence spectra of $\mathrm{TiO}_{2} / \mathrm{Gd}_{2} \mathrm{O}_{2} \mathrm{~S}$ : Tb and PTCDI-modified $\mathrm{TiO}_{2}$ under excitation at $254 \mathrm{~nm}$. Definitions and the correlation of the photocatalytic activity of $\mathrm{TiO}_{2} / \mathrm{Gd}_{2} \mathrm{O}_{2} \mathrm{~S}$ : Tb with its photoluminescence spectra are given in the previous study. ${ }^{14}$ The peak of the photoluminescence spectra of $\mathrm{TiO}_{2}(\mathrm{P} 25)$ is located near $490 \mathrm{~nm}$; the PTCDI peak, including the lower shoulder peak, is located near $695 \mathrm{~nm}$. The detected emission peak in the longer wavelength corresponds to the vibration modes in PTCDI $\left(\sim 1340-1790 \mathrm{~cm}^{-1}\right)$. This means PTCDI modification was carried out successfully and it enhanced photocatalytic activity. ${ }^{17}$ The shape of the photoluminescence spectrum of PTCDI is very similar to the shape of the UV-vis spectrum. The main peak and lower shoulder peak 


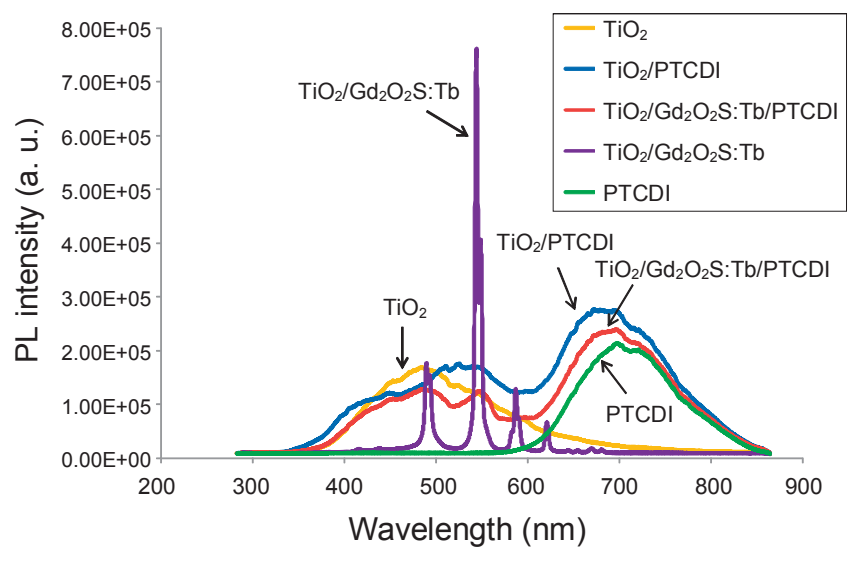

Figure 4. Photoluminescence spectra of $\mathrm{TiO}_{2}(\mathrm{P} 25) / 3,4,9,10$-perylenetetracarboxylic diimide modified $\mathrm{TiO}_{2} / 3,4,9,10$-perylenetetracarboxylic diimide and $\mathrm{Gd}_{2} \mathrm{O}_{2} \mathrm{~S}$ : Tb modified $\mathrm{TiO}_{2} / \mathrm{Gd}_{2} \mathrm{O}_{2} \mathrm{~S}$ : Tb modified $\mathrm{TiO}_{2} / 3,4,9,10$-perylenetetracarboxylic diimide (PTCDI) $(\mathrm{T}=\mathrm{R} . \mathrm{T}$., s.r $\left.=10 \mathrm{sec}^{-1}\right)$.

of the photoluminescence spectra of PTCDI-modified $\mathrm{TiO}_{2}$ were detected at $695 \mathrm{~nm}$ and $717 \mathrm{~nm}$ by comparing the PTCDI spectra with that of $\mathrm{TiO}_{2}(\mathrm{P} 25)$. This means that PTCDI-modified $\mathrm{TiO}_{2}$ absorbs visible light and emits light in the longer wavelength of $695 \mathrm{~nm}$. This shows that its photocatalytic activity as a visible-light harvesting photocatalyst improved. In the photoluminescence spectra of PTCDI-modified $\mathrm{TiO}_{2} / \mathrm{Gd}_{2} \mathrm{O}_{2} \mathrm{~S}: \mathrm{Tb}$, the PTCDI peak is high near $695 \mathrm{~nm}$ compared with that of $\mathrm{TiO}_{2} / \mathrm{Gd}_{2} \mathrm{O}_{2} \mathrm{~S}: \mathrm{Tb}$, which is nearly flat. Furthermore, the photoluminescence spectra of PTCDI-modified $\mathrm{TiO}_{2} / \mathrm{Gd}_{2} \mathrm{O}_{2} \mathrm{~S}: \mathrm{Tb}$ include a peak at $480 \mathrm{~nm}$ and at $545 \mathrm{~nm}$. The highest photocatalytic activity $(59.02 \pm 0.31 \%$, Table 2$)$ shows the modifying effect of PTCDI and $\mathrm{Gd}_{2} \mathrm{O}_{2} \mathrm{~S}$ :Tb. Overall, the results in Table 2 show that photocatalytic activity can be greatly increased by PTCDI and metal oxide.

\section{Conclusions}

We have shown here that $\mathrm{TiO}_{2}$ sensitized with the dye 3,4, 9,10-Perylenetetracarboxylic diimide out performs $\mathrm{TiO}_{2}(\mathrm{De}-$ gussa, P25) in photocatalytic activity and also acts as a visiblelight harvesting photocatalyst.

The case of $\mathrm{TiO}_{2}$ modified with $\mathrm{Gd}_{2} \mathrm{O}_{2} \mathrm{~S}$ : Tb and then further modified with 3,4,9,10-Perylenetetracarboxylic diimide illustrates the gains in photocatalytic activity achieved by this process. This study points the way to the commercialization of a light-harvesting photocatalyst through a thin film optimized for photocatalytic activity and transmittance.

The UV-vis absorption spectra of PTCDI were observed in the wide visible-light region ( $400 \mathrm{~nm}-650 \mathrm{~nm}$ ). Its main peak and lower shoulder peak were observed at $695 \mathrm{~nm}$ and $717 \mathrm{~nm}$ in the photoluminescence spectra. These results support our conclusion that PTCDI improves the photocatalytic activity of semiconductor materials, resulting in a visible-light harvesting photocatalyst. Also, we confirmed that UV pre-treatment enhances photocatalytic activity. UV pre-treatment caused drastic changes on the thin film surface, subsequent to which the thin film exhibited super hydrophilicity, a property that has a large positive effect on photocatalytic activity. However, in one case that deviated from the observed trend, the activity of the $\mathrm{Gd}_{2} \mathrm{O}_{2} \mathrm{~S}$ : Tb-modified $\mathrm{TiO}_{2}$ decreased after UV light irradiation. Perhaps, this is related to disturbances created by the interaction between characteristic changes in the thin film surface and the optical properties of $\mathrm{Gd}_{2} \mathrm{O}_{2} \mathrm{~S}$ : Tb. Further study is needed to explain this apparent anomaly.

Acknowledgments. The author would like to thank KAST (Kanagawa Academy of Science and Technology) photocatalyst laboratory researcher for help and guidance during 2010.01.18 2010.01.29 and also much useful information.

\section{Reference}

1. Fujishima, A.; Rao, T. N.; Tryk, D. A. J. Photochem. Photobiol. $C$ 2000, 1,1 .

2. Minabe, T.; Tryk, D. A.; Sawunyama, P.; Kikuchi, Y.; Hashimoto, K.; Fujishima, A. J. Photochem. Photobiol. A 2000, 137, 53.

3. Nguyen, V. N. H.; Amal, R.; Beydoun, D. Chem. Eng. Sci. 2003, 58,4429 .

4. Colón, G. et al., J. Photochem. Photobiol. A 2006, 179, 20.

5. Morikawa, T.; Irokawa, Y.; Ohwaki, T. Appl. Catal. A 2006, 314 , 123.

6. Tryba, B. Int. J. Photoenergy. 2008, 2008, 15.

7. Ding, H.; Sun, H.; Shan, Y. J. Photochem. Photobiol. A 2005, 169, 101.

8. Ajito, K.; Sukamto, J. P. H.; Nagahara, L. A.; Hashimoto, K.; Fujishima, A. J. Electroanal. Chem. 1995, 386, 229.

9. Zhang, X.; Udagawa, K.; Liu, Z.; Nishimoto, S.; Xu, C.; Liu, Y.; Sakai, H.; Abe, M.; Murakami, T.; Fujishma, A. J. Photochem. Photobiol. A 2009, 202, 39.

10. Li, D.; Huang, H.; Chen, X.; Chen, Z.; Li, W.; Ye, D.; Fu, X. J. Solid State Chem. 2007, 180, 2630.

11. Irie, H.; Watanabe, Y.; Hashimoto, K. J. Phys. Chem. B 2003, 107, 5483.

12. Brochsztain, S. et al., Microporous Mesoporous Mater. 2007, 102, 258.

13. Ko, H. C.; Kim, S.; Choi, W.; Moon, B.; Lee, H. Chem. Commun. 2006, 69.

14. Kim, B. G.; Lee, H. G.; Kim, H. S.; Kim, Y. S. Bull. Korean. Chem. Soc. 2009, 30, 675.

15. Liu, B.; Wen, L.; Zhao, X. Appl. Surf. Sci. 2008, 255, 2752.

16. Janssen, R. A. J. et al., J. Phys. Chem. A 2008, 112, 5846.

17. Djurisic, A. B. et al., Adv. Funct. Mater. 2008, 18, 566. 